\title{
Théologiques
}

\section{Politique et religion au Proche-Orient}

\section{Éli Barnavi}

Volume 11, numéro 1-2, automne 2003

Juifs et chrétiens. L’à-venir du dialogue.

URI : https://id.erudit.org/iderudit/009533ar

DOI : https://doi.org/10.7202/009533ar

Aller au sommaire du numéro

Éditeur(s)

Faculté de théologie de l'Université de Montréal

ISSN

1188-7109 (imprimé)

1492-1413 (numérique)

Découvrir la revue

Citer cet article

Barnavi, É. (2003). Politique et religion au Proche-Orient. Théologiques, 11(1-2), 223-231. https://doi.org/10.7202/009533ar d'utilisation que vous pouvez consulter en ligne.

https://apropos.erudit.org/fr/usagers/politique-dutilisation/ 


\title{
Politique et religion au Proche-Orient
}

\author{
Éli BARNAVI \\ Département d'histoire \\ de l'Occident moderne \\ Université de Tel Aviv
}

Il est banal de constater qu'au Proche-Orient la politique entretient avec la religion des rapports complexes, confus et plutôt conflictuels ${ }^{1}$. C'est du moins la conception libérale dominante en Occident, selon laquelle ces deux entités ne font décidément pas bon ménage. La religion, détentrice de la Vérité, est le domaine de l'absolu; la politique, pourvoyeuse de vérités multiples, est celui du relatif. Celle-là prétend montrer la voie du salut éternel, celle-ci ne cherche qu'à guider les hommes sur les chemins incertains d'ici-bas. La laïcité, c'est précisément le refus de confondre ces deux domaines, en refoulant le premier dans la sphère du privé et en réservant le second aux seuls intérêts profanes de l'individu et de la collectivité.

Habitué depuis des siècles à penser ces catégories comme des entités rigoureusement distinctes, puis séparées, l'Occidental a tendance à scruter le contenu théologique des systèmes religieux afin de comprendre pourquoi tous ne fonctionnent pas de la même manière dans une situation donnée. Il cherche Ben Laden dans le Coran, les colons exaltés des Territoires occupés dans la Bible et, accessoirement, les fondamentalistes chrétiens de la Bible Belt américaine dans les Évangiles. Il a tort. Les textes sacrés n'expliquent rien, pour la bonne raison qu'ils disent tout. Aussi y trouve-t-on ce qu'on y cherche. Pour ne prendre qu'un exemple, en Israël, où la Bible est le grand livre de référence de tout le monde,

1. Texte remanié d'une conférence prononcée à l'Université de Montréal, le 17 septembre 2003 . 
religieux comme laïcs, droite et gauche, modérés et jusqu'au-boutistes, les partisans d'une paix de compromis avec les Palestiniens citeront plus volontiers les Prophètes, les tenants du Grand Israël, le livre de Josué. Un pamphlet ligueur dans la France des Guerres de religion a dit cela très bien: "L'Évangile est couteau de fripière, qui tranche des deux côtés."

C'est une question de bon sens historique: les textes ont beau être les mêmes, leur lecture est différente, car tout est dans l'interprétation. L'islam? Quel islam? Celui des Omeyyades ou celui des Almohades, celui d'Abdallah de Jordanie ou celui d'Abdallah d'Arabie, celui des soufis ou celui des Wahhabites? Le christianisme? Quel christianisme? Celui des mystiques ou celui des inquisiteurs, celui des massacreurs de la SaintBarthélemy ou celui d'Henri IV, celui de monseigneur Lefèvre ou celui de Jean XXIII ? Le judaïsme, quel judaïsme? Celui d'Yitzhak Rabin ou celui de son assassin? C'est dire que ce qui est pertinent pour comprendre le comportement des fidèles d'une religion est plutôt la manière dont cette religion fonctionne en tant qu'institution sociale et politique dans un contexte historique donné, la manière dont elle est au monde, ici et maintenant.

Cela dit, les rapports entre politique et religion ne sont pas les mêmes dans tous les systèmes religieux. Dans le christianisme, la distinction entre les deux «royaumes" a été posée d'emblée: Jésus n'annonce-t-il pas que le sien n'est pas de ce monde, n'affirme-t-il point qu'il faut rendre à Dieu ce qui est à Dieu et à César ce qui est à César ? C'est que les adeptes de la nouvelle foi agissent dans le cadre préétabli de l'Empire romain, et qu'ils s'y adaptent sans tenter de le supplanter. Au contraire, l'Église, qui s'installe à Rome plutôt qu'à Jérusalem, se coule dans les cadres de l'Empire, dont elle se veut l'héritière. Les deux épées, celle, spirituelle, du pape et celle, temporelle, de l'empereur, ne se confondront jamais: alliées en principe, conflictuelles en fait, elles resteront toujours des armes distinctes, soigneusement séparées. Et, lorsque l'État territorial moderne émergera sur les ruines d'un Saint Empire romain vidé de sa substance, la tentation théocratique des papes sera tenue en échec par les rois, réputés "empereurs en leur royaume», c'est-à-dire souverains. En fait, l'État moderne ne pouvait être que laïque. Lorsque Machiavel décrit un domaine de la politique a-thée au sens premier du terme, arène d'un jeu de pouvoir où seuls comptent les intérêts et les passions des hommes, il ne fait que théoriser une situation de fait. En un sens, le christianisme est laïque par définition. 
Bien entendu, distinction n'est pas séparation, et tous les États de la chrétienté ne sont pas parvenus à la séparation de l’Église et de l'État en suivant la même voie. Dans les pays protestants, où la révolution politique - aux Pays-Bas au Xvi ${ }^{\mathrm{e}}$ siècle, en Angleterre au XVII ${ }^{\mathrm{e}}$, en Amérique du Nord un siècle plus tard — s'est faite au nom de la Bible, la séparation a été sans douleur. En France, où la Réforme a échoué et l'Église s'est montrée hostile au changement, la Révolution a pris un tour franchement anticlérical, voire antichrétien, et la séparation a été un combat. Mais dans tous les cas de figure, la séparation était inscrite dans la logique de la distinction et, quelles qu'en fussent les modalités, le résultat a été le même partout.

La situation est très différente dans les deux autres monothéismes. Dans l'islam, la révélation du Prophète a précédé l'État, qui a été créé après coup, à la pointe de l'épée, au nom des principes de la nouvelle foi. Dans le judaïsme, religion traditionnellement dépourvue d'État, le caractère ethnique de la religion interdit en principe la séparation radicale entre deux domaines étroitement imbriqués, dont nul ne peut définir une véritable ligne de partage. Dans les deux cas, on a affaire à une impossible laïcité.

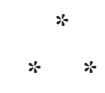

Avec tout cela à l'esprit, abordons maintenant les rapports entre politique et religion dans le Proche-Orient actuel. Sauf au Liban, pays d'un improbable équilibre confessionnel, l'islam y est partout religion d'État. Entre l'État — produit d'importation mal intégré dans la culture locale - et la religion - largement dominée par une lecture politique jusqu'au-boutiste de l'islam - s'est instaurée une sorte d'équilibre de la terreur. Tout s'y passe comme si l'État, plus ou moins absolutiste, avait passé avec les intégristes un marché tacite: la paix civile (relative) et la perpétuation du pouvoir, en échange d'un droit de regard sur la discipline morale des masses et la libre expression du seul rejet haineux des ennemis intérieurs (libéraux, homosexuels, chrétiens) et extérieurs (Juifs, Américains, Occidentaux).

En effet, la caractéristique principale du Machrek, comme du Maghreb d'ailleurs, est la montée de l'intégrisme. Je dis bien intégrisme, et non fondamentalisme, qui me paraît relever d'une logique différente. 
Le fondamentaliste est celui qui prétend faire retour vers les «fondements » de la foi en collant d'aussi près que possible aux préceptes de la religion et parfois, mais pas toujours, en se livrant à une lecture littérale des textes sacrés. Ainsi, un ultra-orthodoxe juif est un fondamentaliste, mais pas nécessairement un intégriste. L'intégrisme, lui, est toujours politique.

Qu'est-ce donc que l'intégrisme? Je vois trois définitions possibles, qui se recoupent: la religion faite politique radicale; l'investissement de l'ensemble du champ politique par la religion; la religion qui fonctionne comme idéologie totale (ce qui la rapproche des religions séculières de substitution, comme le communisme ou le fascisme).

Les causes de l'émergence de l'islam intégriste sont partout les mêmes. Deux me paraissent essentielles: l'incapacité que j'évoquais auparavant à concevoir un domaine politique propre, indépendant de Dieu; et l'incapacité à se mesurer aux défis de la modernité. L'analyse des raisons de ce ratage, par une civilisation jadis glorieuse, d'une modernité imposée par l'Occident n'est pas de notre ressort ici. Elles sont d'ailleurs loin de faire l'unanimité parmi les spécialistes. Si, comme je le pense, le grand secret de l'Occident chrétien est la laïcité, alors la confusion du spirituel et du temporel et la sujétion de celui-ci à celui-là, constitue la cause première du retard de l'Orient musulman. Pour ne prendre qu'un exemple, mais combien révélateur, il y eut des presses juives et chrétiennes à Istanbul dès la fin du $\mathrm{Xv}^{\mathrm{e}}$ siècle, mais ce n'est que deux siècles plus tard qu'une presse musulmane put y être installée contre l'opposition farouche des ulémas. Aujourd'hui il y a, bien sûr, des presses en nombre dans tous les pays de l'islam. Mais deux rapports consécutifs du Programme de l'ONU pour le développement, rédigés d'ailleurs par des Arabes, nous apprennent que l'ensemble du monde arabo-musulman a traduit en mille ans moins d'ouvrages étrangers que l'Espagne n'en traduit en une seule année et qu'aujourd'hui, 260 millions d'Arabes disposent de moins de livres traduits en leur langue que 11 millions de Grecs...

Ce qui est certain est que, dans le bras de fer qui l'oppose dès l'origine à l'Occident chrétien, le monde arabo-musulman a perdu depuis fort longtemps la partie, et que l'écart ne cesse de se creuser tragiquement. Or, sous l'influence des puissances coloniales ou, après l'accession à l'indépendance, par libre choix de leurs élites, les pays de l'islam ont essayé tous les ismes venus d'Occident, du libéralisme au socialisme en passant par le nationalisme, local ou panarabe. Ils ont tout essayé, rien n'a 
marché. C'est ainsi que s'explique le choix intégriste. Le raisonnement est simple: non seulement l'imitation servile de modèles étrangers n'a-t-elle servi à rien, elle a aggravé la misère et l'humiliation. Il faut donc revenir à la seule valeur qui appartient en propre aux musulmans, et qui a fait autrefois leur splendeur: l'islam. Soit dit en passant, le «retour» à un ordre ancien est toujours du domaine de l'illusion pure. Non seulement on ne revient jamais en arrière - le monde disparu étant à jamais englouti dans l'épaisseur d'une histoire qui ne cesse de se renouveler mais encore l'intégrisme, tel qu'il se présente aujourd'hui, dans cette formidable coexistence d'une pensée archaïque avec une organisation et des moyens hi tech, est lui-même un phénomène essentiellement moderne. Il n'y a rien dans l'Iran médiéval qui préfigure la constitution de la République des ayatollahs, ni dans l'islam classique qui rende compte d'une organisation comme al-Qaïda.

Quoi qu'il en soit, dans le contexte du conflit israélo-arabe, l'islam politique semble le seul moyen de combattre efficacement Israël. En chassant Israël du Sud-Liban, le Hezbollah libanais s'est glorifié d'avoir fait ce qu'aucune armée arabe n'avait réussi à faire: contraindre Tsahal à reculer. Et l'attentat-suicide pratiqué par le Hamas et le Djihad islamique s'est révélé être une arme d'une redoutable efficacité. Mais, contrairement à une idée bien ancrée en Occident, la lutte contre Israël n'est pas l'essentiel: le projet islamiste est global. Ainsi, en confondant causes et conséquences, on s'imagine volontiers que le président Sadate a été assassiné par des officiers islamistes parce qu'il avait fait la paix avec Israël. En fait, la première raison invoquée par le chef des conjurés fut... l'interdiction du voile à l'université. Sadate a été assassiné parce qu'il était le chef impie d'un État impie. Et, bien entendu, un tel État ne pouvait que faire la paix avec l'ennemi sioniste...

Ce projet global - une guerre apocalyptique contre «les Juifs et les croisés ", dont l'objet est de repousser aussi loin que possible les limites du dar al-islam - est évident dans le cas d'al-Qaïda. En effet, l'organisation d'Ossama ben Laden a pris corps dans la foulée de la première guerre du Golfe dans le but de chasser les infidèles de la terre sacrée d'Arabie. Mais la libération des lieux saints de l'islam n'est qu'une étape dans un très long chemin. Dans son recrutement comme dans ses objectifs, la «Base» est une internationale. Mais même les mouvements d'apparence nationale, comme le Hamas et le Djihad islamique palestiniens et le Hezbollah libanais, ne considèrent leur combat contre Israël que 
comme un épisode d'une entreprise beaucoup plus vaste, une Palestine musulmane sur l'ensemble du territoire de la mer au Jourdain devant s'insérer à terme dans une oumma (communauté des fidèles) reconstituée.

Israël n'a pas échappé à l'intégrisme non plus. Pour une part, l'intégrisme juif relève du même phénomène de rejet de la modernité et de nostalgie pour un monde disparu que nous avons observé dans l'islam. C'est le cas, par exemple, du parti Shass, un décalque «oriental» de l'ultra-orthodoxie ashkénaze, qui prétend représenter le judaïsme traditionnel séfarade. C'est le cas aussi d'une frange extrême du camp national-religieux, sur laquelle je reviendrai. Cela dit, l'intégrisme juif présente tout de même des traits de caractère particuliers. Israël étant une société du premier monde industrialisé, peu propice par définition à une implantation forte du phénomène intégriste, celui-ci est nécessairement ultra-minoritaire. Par ailleurs, il s'agit d'un «intégrisme dans un seul pays », le judaïsme, religion ethnique, on l'a vu, ne cherchant pas à faire des prosélytes et ne s'exportant donc pas aux quatre coins de la planète, sous les auspices de l'intégrisme ou autrement.

Aux racines de la principale composante de l'intégrisme israélien la mouvance Grand Israël -, il y a une lecture particulière du sionisme politique. On sait que le gros de l'orthodoxie juive a été d'emblée très hostile au sionisme. Pour le judaïsme orthodoxe, l'exil est une punition divine, et seule la volonté divine, manifestée par l'apparition du Messie, est susceptible de ramener le peuple d'Israël vers la Terre promise. Or, le sionisme, mouvement laïque et émancipateur inspiré par le mouvement des nationalités européen, entendait organiser ces retrouvailles du peuple et de la Terre par des moyens terrestres - autrement dit, par un mouvement politique de masse. Cependant, pour des raisons à la fois pratiques - la situation désespérée des masses juives en Europe orientale et idéologiques, une frange très minoritaire de l'orthodoxie a tout de même fait exception à la règle et a rejoint le mouvement sioniste: ce fut le mouvement Mizrahi, créé à la fin du $\mathrm{XIX}^{\mathrm{e}}$ siècle et devenu après la création de l'État d'Israël le Parti national-religieux. Formation très modérée durant les 30 premières années de l'État, le PNR a loyalement servi son grand frère travailliste, en se fixant comme seule politique le 
maintien d'un caractère aussi «juif » que possible, c'est-à-dire, dans son esprit, aussi religieux que possible, à l'État d'Israël. C'est après la guerre des Six-Jours et le choc provoqué par les retrouvailles jugées miraculeuses avec la vieille ville de Jérusalem et la Judée-Samarie - le cœur de l'Israël biblique - que le Parti national-religieux devait changer de nature. Issue des yeshivoth (académies talmudiques) affiliées à ce parti, une nouvelle génération de chefs, très différente des caciques prudents de la vielle école, s'est imposée peu à peu. Puis, dans la foulée de la guerre du Kippour, a fait son apparition son émanation extra-parlementaire: le Goush Emounim (Bloc de la foi), fer de lance de la colonisation des territoires occupés lors de la campagne éclair de juin 1967. Une nouvelle idéologie a investi les rangs du camp national-religieux: un néosionisme d'essence messianique, qui a renversé la perspective du sionisme originel. Celui-ci entendait remettre les Juifs dans l'histoire en normalisant leur existence nationale et leurs relations avec les Gentils; pour les tenants de ce nouvel intégrisme juif, inédit dans le paysage politique israélien, il s'agissait plutôt de pérenniser l'exception juive en l'ancrant dans la Terre retrouvée. Pour le sionisme classique, c'est le peuple juif qu'il fallait sauver; pour les néosionistes messianiques, c'est plutôt la Terre.

Or, si elle est restée très minoritaire, cette branche tard venue de la famille sioniste a fini par intimider l'ensemble de la classe politique et dicter l'agenda national. Comment cela a-t-il été possible? Par un phénomène somme toute fréquent dans l'histoire politique. Pour l'essentiel, le néosionisme religieux s'est engouffré dans le vide idéologique laissé par un sionisme laïque en bout de course, vidé de sa substance par son succès même. Toute idéologie qui atteint son objectif — en l'occurrence, la création d'un État juif - s'essouffle, dégénère en un chapelet de slogans creux et finit par s'effacer devant la vigueur nouvelle d'une idéologie à la fois proche et concurrente. Le sionisme a suivi la pente commune.

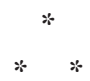

Risquons en conclusion un coup d'œil vers l'avenir. Si l'on est pessimiste, on découvrira un paysage bien sombre. La guerre contre l'intégrisme musulman ne fait que commencer - une guerre sournoise, sale, où l'ennemi est insaisissable et la ligne de front invisible. Mais si la ligne de front n'est pas visible, elle n'en est pas moins réelle. Il y en a même deux: une 
mondiale, planétaire; et une qui passe à l'intérieur des sociétés, aussi bien en Orient qu'en Occident. Cette guerre entre deux conceptions de l'homme, de la société, de la place de l'homme dans la société, cette guerre de civilisations en somme, va affecter de plus en plus notre niveau de vie, notre vision du monde et nos libertés. De plus, son issue n'est pas certaine, et elle risque de déboucher sur des catastrophes inouïes, dont la double attaque du 11 septembre n'offre qu'une pâle préfiguration.

Si l'on est d'humeur optimiste, on aura une vision beaucoup plus apaisée. La crête de la vague intégriste est derrière nous. En Occident, la "sortie du religieux" théorisée par Marcel Gauchet se poursuit sans désemparer. En pays d'islam, non seulement l'État reste puissant, comme l'a amplement démontré le cas algérien; mais encore, et surtout, l'expérience iranienne constitue la preuve irréfutable que l'intégrisme n'est pas la solution aux maux dont souffre la société musulmane. Par ailleurs, la modernité conserve partout une formidable force d'attraction. Enfin, parmi les Israéliens le rêve du Grand Israël est virtuellement fini, et le néosionisme messianique que j'ai évoqué donne à son tour des signes évidents d'essoufflement. Le règlement du contentieux palestinien ne mettra certes pas fin à l'intégrisme islamiste; mais il lui enlèvera un prétexte majeur, tout en tarissant considérablement ses sources de recrutement.

Je suis bien sûr incapable de trancher entre ces deux versions, toutes deux plausibles, d'un avenir impénétrable par définition. On sait que le Talmud réserve le don prophétie depuis la chute du Temple aux enfants et aux fous. Mais je crois savoir comment il faut combattre ce fascisme $\mathrm{du} \mathrm{XXI}^{\mathrm{e}}$ siècle: militairement, sans faiblesse, mais aussi en s'attaquant à ses principales causes - la misère, le sous-développement, l'ignorance. Et de plus, sans doute, en adaptant la laïcité aux besoins de notre temps: une laïcité moins crispée, ferme sur les principes mais ouverte sur le savoir. 


\section{RÉSUMÉ}

Les trois religions monothéistes n'articulent pas de la même manière politique et religion. Dans ce cadre général, le cas du Proche-Orient actuel est particulier. Comment expliquer la montée de l'intégrisme qu'on y observe, tant en pays musulmans qu'en Israël, sinon par l'échec de projets politiques mobilisateurs? Deux lectures de la confrontation modernité / intégrisme demeurent possibles: une pessimiste, qui entrevoit un conflit tragique entre deux conceptions de l'humain; une optimiste, qui conclut à l'essoufflement de l'intégrisme.

\section{ABSTRACT}

The three monotheistic religions do not articulate politics and religion in the same way. Actually, the Near East seems to be a particular case. How can one explain the growing ultra-conservatism which can be observed in Muslim countries as well as in Israel, if not that the political policies fail to mobilize people? A two way reading of the confrontation between modernity and ultra-conservatism seems possible: a pessimistic view would see here a tragic conflict between two representations of the human being; an optimistic view would conclude that ultra-conservatism is running out of steam. 